\title{
Note on the solution of random differential equations via $\psi$-Hilfer fractional derivative
}

\author{
S. Harikrishnan ${ }^{1}$, Kamal Shah² ${ }^{2 *}$ Dumitru Baleanu ${ }^{3}$ and K. Kanagarajan
}

*Correspondence:
kamalshah408@gmail.com
${ }^{2}$ Department of Mathematics,
University of Malakand, Chakdara,
Pakistan
Full list of author information is
available at the end of the article

\begin{abstract}
This manuscript is devoted to an investigation of the existence, uniqueness and stability of random differential equations with $\psi$-Hilfer fractional derivative. The concerned investigation of existence and uniqueness is obtained via the Schauder fixed point theorem and Banach contraction principle, respectively. Furthermore, for the respective solutions, some results related to different kinds of Ulam type stability including Hyers-Ulam, and generalized Hyers-Ulam, Hyers-Ulam-Rassias are obtained.
\end{abstract}

MSC: $26 \mathrm{~A} 33 ; 34 \mathrm{~B} 10 ; 34 \mathrm{~A} 12$

Keywords: Random differential equations; $\psi$-Hilfer fractional derivative; Existence theory; Stability analysis

\section{Introduction}

In the previous few decades non-integer order differential and integral equations were given much attentions. Because fractional differential equations (FDEs) rise up certainly in various fields which include rheology, fractals, chaotic dynamics, control theory, signal processing, bioengineering and biomedical applications. The theory of FDEs has been extensively studied and developed by many authors, see $[6,9,13]$. The dynamical analysis of FDEs has been investigated very well in last few years, one can see $[2,3,5,7,8,12,20-22$, 24] for details.

Since various real world processes/phenomena have inconsistent dynamical behavior, for instance in a powerful magnetic field communications of signals, diffusion of pollution in atmosphere, traffic networks, in financial markets the effect of speculations on the profitability of stocks, and so on. For the aforesaid processes/phenomena, the traditional models are not suitable to express the characteristics accurately. Therefore in such a situation FDEs play vital roles in describing the aforementioned phenomena more accurately. On the other hand, to describe a real system involving complexities and uncertainties via using deterministic FDEs is not a good choice. On the other hand to bring random factors under consideration, numerous mathematical models of random differential equations (RFDEs) were considered in the last few years. In this regard some very good results were obtained corresponding to mathematical models by using RFDEs; see [15].

(c) The Author(s) 2018. This article is distributed under the terms of the Creative Commons Attribution 4.0 International License (http://creativecommons.org/licenses/by/4.0/), which permits unrestricted use, distribution, and reproduction in any medium, provided you give appropriate credit to the original author(s) and the source, provide a link to the Creative Commons license, and indicate if changes were made. 
In the last few years, many papers relating to RFDEs (stochastic differential equations) were published; see $[1,11,23]$. In fact, RFDEs are the natural extensions of deterministic FDEs. In many applications of real world problems, the use of the aforesaid RFDEs have been considered by many researchers. Motivated by the above works here we discuss the dynamical behavior of RFDEs involving a $\psi$-Hilfer fractional derivative (HFD) of the form

$$
\left\{\begin{array}{l}
D^{\alpha, \beta ; \psi} \mathfrak{h}(t, \vartheta)=\mathfrak{g}(t, \vartheta, \mathfrak{h}(t, \vartheta)), \quad t \in J:=[0, T], T>0 \\
\left.\ell^{1-v ; \psi} \mathfrak{h}(t, \vartheta)\right|_{t=0}=\mu(\vartheta),
\end{array}\right.
$$

where $\mathscr{D}^{\alpha, \beta ; \psi}$ is $\psi$-HFD of order $\alpha \in(0,1)$ and type $\beta \in[0,1]$. Furthermore, $\mathfrak{h}$ is a random function, $\vartheta$ is the random variable and $\ell^{1-v}$ is the $\psi$-fractional integral of order $1-v(v=$ $\alpha+\beta-\alpha \beta)$. Let $\Omega$ be the probability space with a continuous function $\mathfrak{g}: J \times \Omega \times \mathbb{R} \rightarrow \mathbb{R}$ such that $\vartheta \in \Omega$. Thanks to the Schauder fixed point theorem and the Banach contraction principle, some interesting results related to the existence and uniqueness of solutions were established. Furthermore, as stability has become an important aspect in recent times from the optimization and numerical points of view, some results devoted to Hyers-Ulam (HU), Hyers-Ullam-Rassias (HUR) and generalized Hyers-Ulam (GHU) stabilities are also discussed for the considered RFDEs with $\psi$-HFD.

Recently, Almeida [4] using the suggestion of the fractional derivative in the Caputo sense, recommended a new fractional derivative called the $\psi$-Caputo derivative with respect to another function, which simplifies a class of fractional derivatives. These integrals and fractional derivatives have unlike kernels and this leads to a number of definitions. In this viewpoint, we will employ the HFD idea, and we suggest a fractional differential operator of a function with respect to another $\psi$ function, the so-called $\psi$-HFD. With the help of the fractional operator suggested here, we use the freedom of choice of the standard differential operator. Recently, Sousa et al. introduced a recent and interesting definition of fractional integral and some applications. In this regard, there are other stabilizing jobs that are interesting, because such definitions and stability results are indeed important for the development and expansion of the fractional calculation and its applications in various branches of science and technology. Numerous problems which are based on this derivative are considered by many authors, see for instance [17-21] and the references therein.

\section{Preliminaries}

Some basic definitions and results are introduced in the present section; for details see $[1,16]$. Let $C$ be the Banach space of all continuous functions $\mathfrak{g}: J \times \Omega \rightarrow \mathbb{R}$ with the norm $\|\mathfrak{g}\|_{C_{v, \psi}}=\sup \{|\mathfrak{g}(t, \vartheta)|: t \in J\}$. We denote the weighted spaces of all continuous functions defined by $C_{v, \psi}(J, \mathbb{R})=\left\{\mathfrak{g}: J \rightarrow \mathbb{R}:(\psi(t)-\psi(0))^{v} \mathfrak{g}(t) \in C\right\}, 0 \leq v<1$, with the norm $\|\mathfrak{g}\|_{C_{v, \psi}}=\sup _{t \in J}\left|(\psi(t)-\psi(0))^{v} \mathfrak{g}(t)\right|$.

Definition 2.1 ([16]) Corresponding to the function $\psi$ on $J$, the fractional integral of $\mathfrak{h}$ operating from the left side is described as

$$
\left(\ell^{\alpha ; \psi}\right) \mathfrak{h}(t)=\int_{0}^{t} \psi^{\prime}(s) \frac{(\psi(t)-\psi(s))^{\alpha-1}}{\Gamma(\alpha)} \mathfrak{h}(s) d s, \quad t \in(0, T]
$$


Definition 2.2 ([16]) For fractional order $\alpha>0$, the Riemann-Liouville derivative of a function $\mathfrak{h}$ corresponding to another function $\psi$ with $\psi^{\prime}(t) \neq 0$ is described as

$$
\mathscr{D}^{\alpha ; \psi} \mathfrak{h}(t)=\left(\frac{1}{\psi^{\prime}(t)} \frac{d}{d t}\right)^{n} \int_{0}^{t} \psi^{\prime}(s) \frac{(\psi(t)-\psi(s))^{n-\alpha-1}}{\Gamma(n-\alpha)} \mathfrak{h}(s) d s
$$

where $n-1=[\alpha]$.

Definition 2.3 ([16]) If $\alpha>0$, and considering two functions $\mathfrak{h}, \psi \in C^{n}(J, \mathbb{R})$ in which $\psi$ is nondecreasing with $\psi^{\prime}(t) \neq 0$, for all $t \in J$, then the left $\psi$-Caputo derivative is described as

$$
\mathscr{D}^{\alpha ; \psi} \mathfrak{h}(t)=\ell^{n-\alpha ; \psi}\left(\frac{1}{\psi^{\prime}(t)} \frac{d}{d t}\right)^{n} \mathfrak{h}(t)
$$

where $n-1=[\alpha]$.

Definition 2.4 ([16]) The $\psi$-HFD of function $\mathfrak{h}$ of order $\alpha>0$ is provided by

$$
\mathfrak{D}^{\alpha, \beta ; \psi} \mathfrak{h}(t)=\ell^{\beta(1-\alpha) ; \psi}\left(\frac{1}{\psi^{\prime}(t)} \frac{d}{d t}\right) \ell^{(1-\beta)(1-\alpha) ; \psi} \mathfrak{h}(t)
$$

The $\psi$-HFD as defined above can be written in the following form:

$$
\mathfrak{D}^{\alpha, \beta ; \psi} \mathfrak{h}(t)=\mathfrak{l}^{\nu-\alpha ; \psi} \mathfrak{D}^{\nu ; \psi} \mathfrak{h}(t)
$$

To obtain appropriate results about the HU stability and HUR stability for Eq. (1), we describe some inequality in the sequel whose details can be found in $[1,10,25]$.

Let $\epsilon>0$ be a positive real number and $\varphi: J \times \Omega \rightarrow \mathbb{R}^{+}$be a continuous function, then

$$
\begin{aligned}
& \left|\mathcal{D}^{\alpha, \beta ; \psi} \mathfrak{h}(t, \vartheta)-\mathfrak{g}(t, \vartheta, \mathfrak{h}(t, \vartheta))\right| \leq \epsilon, \\
& \left|\mathscr{D}^{\alpha, \beta ; \psi} \mathfrak{h}(t, \vartheta)-\mathfrak{g}(t, \vartheta, \mathfrak{h}(t, \vartheta))\right| \leq \epsilon \varphi(t), \\
& \left|\mathcal{D}^{\alpha, \beta ; \psi} \mathfrak{h}(t, \vartheta)-\mathfrak{g}(t, \vartheta, \mathfrak{h}(t, \vartheta))\right| \leq \varphi(t) .
\end{aligned}
$$

Definition 2.5 Equation (1) is HU stable corresponding to a real number $C_{\mathfrak{g}}>0$ and for every $\epsilon>0$, if for every solution $\mathfrak{h}: \Omega \rightarrow C_{1-v, \psi}$ of the inequality (6) one has a unique solution $\mathfrak{y}: \Omega \rightarrow C_{1-v, \psi}$ of Eq. (1) that satisfies

$$
|\mathfrak{h}(t, \vartheta)-\mathfrak{y}(t, \vartheta)| \leq C_{\mathfrak{g}} \epsilon, \quad t \in J, \vartheta \in \Omega
$$

Definition 2.6 Equation (1) is GHU stable against $\varphi_{\mathfrak{g}} \in C([0, \infty),[0, \infty)), \varphi_{\mathfrak{g}}(0)=0$ if for every solution $\mathfrak{h}: \Omega \rightarrow C_{1-v, \psi}$ of the inequality (6) one has a unique solution $\mathfrak{y}: \Omega \rightarrow C_{1-v, \psi}$ of Eq. (1) that satisfies

$$
|\mathfrak{h}(t, \vartheta)-\mathfrak{y}(t, \vartheta)| \leq \varphi_{\mathfrak{g}} \epsilon, \quad t \in J, \vartheta \in \Omega
$$


Definition 2.7 Equation (1) is HUR stable corresponding to $\varphi$ if we have a real number $C_{\mathfrak{g}, \varphi}>0$ such that for every $\epsilon>0$ and for each solution $\mathfrak{h}: \Omega \rightarrow C_{1-v, \psi}$ of the inequality (7) there exists a unique solution $\mathfrak{y}: \Omega \rightarrow C_{1-v, \psi}$ of Eq. (1) with

$$
|\mathfrak{h}(t, \vartheta)-\mathfrak{y}(t, \vartheta)| \leq C_{\mathfrak{g}, \varphi} \in \varphi(t), \quad t \in J, \vartheta \in \Omega
$$

Definition 2.8 Equation (1) is generalized HUR stable corresponding to a function $\varphi$ with $C_{\mathfrak{g}, \varphi} \in(0, \infty)$, if to every solution $\mathfrak{h}: \Omega \rightarrow C_{1-v, \psi}$ of the inequality (8) there is a unique solution $\mathfrak{y}: \Omega \rightarrow C_{1-v, \psi}$ of Eq. (1) such that

$$
|\mathfrak{h}(t, \vartheta)-\mathfrak{y}(t, \vartheta)| \leq C_{\mathfrak{g}, \varphi} \varphi(t, \vartheta), \quad t \in J, \vartheta \in \Omega
$$

Lemma 2.9 (Grönwall's lemma [19]) Let $\alpha>0, a(t, \vartheta)>0$ be a locally integrable function on $J \times \Omega$ and $\mathfrak{g}(t, \vartheta)$ be a increasing and nonnegative continuous function on $J \times \Omega$, such that $|\mathfrak{g}(t, \vartheta)| \leq K$ for some constant $K$. Moreover, let $\mathfrak{h}(t, \vartheta)$ be a nonnegative locally integrable function on $J \times \Omega$ with

$$
\mathfrak{h}(t, \vartheta) \leq a(t, \vartheta)+g(t, \vartheta) \int_{0}^{t} \psi^{\prime}(s)(\psi(t)-\psi(s))^{\alpha-1} \mathfrak{h}(s, \vartheta) d s, \quad(t, \vartheta) \in J \times \Omega,
$$

with some $\alpha>0$. Then

$$
\begin{aligned}
\mathfrak{h}(t, \vartheta) \leq & a(t, \vartheta) \\
& +\int_{0}^{t}\left[\sum_{n=1}^{\infty} \frac{(\mathfrak{g}(t, \vartheta) \Gamma(\alpha))^{n}}{\Gamma(n \alpha)} \psi^{\prime}(s)(\psi(t)-\psi(s))^{n \alpha-1}\right] a(s, \vartheta) d s, \quad(t, \vartheta) \in J \times \Omega .
\end{aligned}
$$

Theorem 2.10 (Schauder fixed point theorem [14]) Let B be closed, convex and nonempty subset of a Banach space $C$. Let $\mathcal{T}: B \rightarrow B$ be a continuous mapping such that $\mathcal{T}(B)$ is a relatively compact subset of $C$. Then $\mathcal{T}$ has at least one fixed point in $B$.

\section{Main results}

The existence theory of solutions to Eq. (1) is presented in this section.

For our analysis the following hypotheses should be satisfied.

(H1) There exists a Carathèodory function $\ell: J \times \Omega \rightarrow \mathbb{R}$, such that

$$
|\mathfrak{g}(s, \vartheta, \mathfrak{h}(s, \vartheta))-\mathfrak{g}(s, \vartheta, \mathfrak{y}(s, \vartheta))| \leq \ell(t, \vartheta)|\mathfrak{h}-\mathfrak{y}|
$$

for every $t \in J$ and $\vartheta \in \Omega$.

(H2) There exists $\lambda_{\varphi}>0$ such that, for each $t \in J$ and $\vartheta \in \Omega$, we have

$$
\ell^{\alpha ; \psi} \varphi(t, \vartheta) \leq \lambda_{\varphi} \varphi(t, \vartheta)
$$

Lemma 3.1 A function $\mathfrak{h}$ is the solution of Eq. (1), if and only if $\mathfrak{h}$ satisfies the random integral equation

$$
\mathfrak{h}(t, \vartheta)=\frac{\mu(\vartheta)}{\Gamma(v)}(\psi(t)-\psi(0))^{\nu-1}+\frac{1}{\Gamma(\alpha)} \int_{0}^{t} \psi^{\prime}(s)(\psi(t)-\psi(s))^{\alpha-1} \mathfrak{g}(s, \vartheta, \mathfrak{h}(s, \vartheta)) d s
$$


Theorem 3.2 Assume that hypothesis (H1) is satisfied. Then, Eq. (1) has at least one solution.

Proof Consider the operator $\mathcal{T}(\vartheta): \Omega \times C_{1-v, \psi} \rightarrow C_{1-v, \psi}$. Hence $\mathfrak{h}$ is a solution for Eq. (1) if and only if $\mathfrak{h}=(\mathcal{T}(\vartheta)) \mathfrak{h}$, where the equivalent integral Eq. (9) can be written in the operator form

$$
\begin{aligned}
(\mathcal{J}(\vartheta)) \mathfrak{h}= & \frac{\mu(\vartheta)}{\Gamma(v)}(\psi(t)-\psi(0))^{\nu-1} \\
& +\frac{1}{\Gamma(\alpha)} \int_{0}^{t} \psi^{\prime}(s)(\psi(t)-\psi(s))^{\alpha-1} \mathfrak{g}(s, \vartheta, \mathfrak{h}(s, \vartheta)) d s
\end{aligned}
$$

Clearly, the fixed points of the operator $\mathcal{T}$ are solutions of Eq. (1). Set $\tilde{\mathfrak{g}}=\mathfrak{g}(s, \vartheta, 0)$. For any $\mathfrak{h} \in J \times \Omega$, we have

$$
\begin{aligned}
\left|(\mathcal{T}(\vartheta) \mathfrak{h})(t)(\psi(t)-\psi(0))^{1-v}\right| & \frac{|\mu(\vartheta)|}{\Gamma(v)}+\frac{(\psi(t)-\psi(0))^{1-v}}{\Gamma(\alpha)} \int_{0}^{t} \psi^{\prime}(s)(\psi(t)-\psi(s))^{\alpha-1}|\mathfrak{g}(s, \vartheta, \mathfrak{h}(s, \vartheta))| d s \\
\leq & \frac{|\mu(\vartheta)|}{\Gamma(v)} \\
& +\frac{(\psi(t)-\psi(0))^{1-v}}{\Gamma(\alpha)} \\
& \times \int_{0}^{t} \psi^{\prime}(s)(\psi(t)-\psi(s))^{\alpha-1}|\mathfrak{g}(s, \vartheta, \mathfrak{h}(s, \vartheta))-\mathfrak{g}(s, \vartheta, 0)+\mathfrak{g}(s, \vartheta, 0)| d s \\
\leq & \frac{|\mu(\vartheta)|}{\Gamma(v)} \\
& +\frac{(\psi(t)-\psi(0))^{1-v}}{\Gamma(\alpha)} \\
& \times \int_{0}^{t} \psi^{\prime}(s)(\psi(t)-\psi(s))^{\alpha-1}|\mathfrak{g}(s, \vartheta, \mathfrak{h}(s, \vartheta))-\mathfrak{g}(s, \vartheta, 0)|+|\mathfrak{g}(s, \vartheta, 0)| d s \\
\leq & \frac{|\mu(\vartheta)|}{\Gamma(v)}+\frac{(\psi(t)-\psi(0))^{1-v}}{\Gamma(\alpha)} \ell(t, \vartheta) B(v, \alpha)(\psi(t)-\psi(0))^{\alpha+\nu-1}\|\mathfrak{h}\|_{C_{1-v, \psi}} \\
& +\frac{(\psi(t)-\psi(0))^{1-v}}{\Gamma(\alpha)} B(v, \alpha)(\psi(t)-\psi(0))^{\alpha+\nu-1}\|\tilde{\mathfrak{g}}\|_{C_{1-v, \psi}} \\
\leq & \frac{|\mu(\vartheta)|}{\Gamma(v)}+\frac{\ell(t, \vartheta)}{\Gamma(\alpha)} B(v, \alpha)(\psi(T)-\psi(0))^{\alpha}\|\mathfrak{h}\|_{C_{1-v, \psi}} \\
& +\frac{1}{\Gamma(\alpha)} B(v, \alpha)(\psi(T)-\psi(0))^{\alpha}\|\tilde{\mathfrak{g}}\|_{C_{1-v, \psi}} \\
= & r .
\end{aligned}
$$

This proves that $\mathcal{T}$ transforms the ball $B_{r}=\left\{\mathfrak{h} \in C_{1-v, \psi}:\|\mathfrak{h}\|_{C_{1-v, \psi}} \leq r\right\}$ into itself. We shall show that the operator $\mathcal{T}: B_{r} \rightarrow B_{r}$ satisfies all the conditions of the Schauder fixed point theorem. The proof will be given in the following steps.

Step 1: $\mathcal{T}$ is continuous. 
Let $\mathfrak{h}_{n}$ be a sequence such that $\mathfrak{h}_{n} \rightarrow \mathfrak{h}$ in $C_{1-v, \psi}$. Then for each $t \in J, \vartheta \in \Omega$,

$$
\begin{aligned}
& \left|\left(\left(\mathcal{T}(\vartheta) \mathfrak{h}_{n}\right)(t)-(\mathcal{T}(\vartheta) \mathfrak{h})(t)\right)(\psi(t)-\psi(0))^{1-\nu}\right| \\
& \quad \leq \frac{(\psi(t)-\psi(0))^{1-\nu}}{\Gamma(\alpha)} \int_{0}^{t} \psi^{\prime}(s)(\psi(t)-\psi(s))^{\alpha-1}\left|\mathfrak{g}_{n}(s, \vartheta, \mathfrak{h}(s, \vartheta))-\mathfrak{g}(s, \vartheta, \mathfrak{h}(s, \vartheta))\right| d s \\
& \quad \leq(\psi(t)-\psi(0))^{1-\nu} \frac{B(v, \alpha)}{\Gamma(\alpha)}(\psi(t)-\psi(0))^{\alpha+\nu-1}\left\|\mathfrak{g}_{n}(\cdot, \vartheta, \mathfrak{h}(\cdot, \vartheta))-\mathfrak{g}(\cdot, \vartheta, \mathfrak{h}(\cdot, \vartheta))\right\|_{C_{1-v, \psi}} \\
& \quad \leq \frac{B(v, \alpha)}{\Gamma(\alpha)}(\psi(T)-\psi(0))^{1-v}\left\|\mathfrak{g}_{n}(\cdot, \vartheta, \mathfrak{h}(\cdot, \vartheta))-\mathfrak{g}(\cdot, \vartheta, \mathfrak{h}(\cdot, \vartheta))\right\|_{C_{1-v, \psi}}
\end{aligned}
$$

where $B(v, \alpha)$ is the Beta function. Due to continuity of $\mathfrak{g}$, we have

$$
\left\|\mathcal{T}(\vartheta) \mathfrak{h}_{n}-\mathcal{T}(\vartheta) \mathfrak{h}\right\|_{C_{1-v, \psi}} \rightarrow 0 \quad \text { as } n \rightarrow \infty .
$$

Step 2: $\mathcal{T}\left(B_{r}\right)$ is uniformly bounded.

This is clear since $\mathcal{T}\left(B_{r}\right) \subset B_{r}$ is bounded.

Step 3: We show that $\mathcal{T}\left(B_{r}\right)$ is equi-continuous.

Let $t_{1}>t_{2} \in J$ with $B_{r}$ a bounded set of $C_{1-v, \psi}$ as in Claim 2, and $\mathfrak{h} \in B_{r}$. Then

$$
\begin{aligned}
& \left|\left(\psi\left(t_{1}\right)-\psi(0)\right)^{1-v} \mathcal{T}(\vartheta) \mathfrak{h}\left(t_{1}\right)-\left(\psi\left(t_{2}\right)-\psi(0)\right)^{1-v} \mathcal{T}(\vartheta) \mathfrak{h}\left(t_{2}\right)\right| \\
& \leq \mid \frac{\left(\psi\left(t_{1}\right)-\psi(0)\right)^{1-v}}{\Gamma(\alpha)} \int_{0}^{t_{1}} \psi^{\prime}(s)\left(\psi\left(t_{1}\right)-\psi(s)\right)^{\alpha-1} \mathfrak{g}(s, \vartheta, \mathfrak{h}(s, \vartheta)) d s \\
& \quad-\frac{\left(\psi\left(t_{2}\right)-\psi(0)\right)^{1-\nu}}{\Gamma(\alpha)} \int_{0}^{t_{2}} \psi^{\prime}(s)\left(\psi\left(t_{2}\right)-\psi(s)\right)^{\alpha-1} \mathfrak{g}(s, \vartheta, \mathfrak{h}(s, \vartheta)) d s \mid \\
& \leq \frac{\|\mathfrak{g}\|_{C_{1-v, \psi}}}{\Gamma(\alpha)} B(v, \alpha)\left|\left(\psi\left(t_{1}\right)-\psi(0)\right)^{\alpha}-\left(\psi\left(t_{2}\right)-\psi(0)\right)^{\alpha}\right| .
\end{aligned}
$$

Keeping in mind that upon using $t_{1} \rightarrow t_{2}$ the right hand side of the inequality (11) approaches zero. Therefore by Steps 1-3 together with the Arzela-Ascoli theorem, we see that $\mathcal{T}$ is continuous and compact. Hence by Schauder's theorem, the operator $\mathcal{T}$ has a fixed point $\mathfrak{h}$ which is a solution of Eq. (1).

Lemma 3.3 Assume that the hypothesis (H1) is satisfied. If

$$
\frac{\ell(t, \vartheta)}{\Gamma(\alpha)}(\psi(T)-\psi(0))^{\alpha} B(v, \alpha)<1
$$

Then, (1) has unique solution.

Now we are concerned with the generalized HUR stablest of Eq. (1).

Theorem 3.4 Under the hypotheses (H1) and (H2) the solution of Eq. (1) is generalized HUR stable.

Proof Assume that for any solution $\mathfrak{h}$ of inequality (8) and using Lemma 3.3 one has a unique solution $\mathfrak{y}$ for Eq. (1). Thus we have

$$
\mathfrak{y}(t, \vartheta)=\frac{\mu(\vartheta)}{\Gamma(v)}(\psi(t)-\psi(0))^{\nu-1}+\frac{1}{\Gamma(\alpha)} \int_{0}^{t} \psi^{\prime}(s)(\psi(t)-\psi(s))^{\alpha-1} \mathfrak{g}(s, \vartheta, \mathfrak{y}(s, \vartheta)) d s .
$$


By differentiating inequality (8) for each $t \in J, \vartheta \in \Omega$, we have

$$
\begin{aligned}
& \left|\mathfrak{h}(t, \vartheta)-\frac{\mu(\vartheta)}{\Gamma(\nu)}(\psi(t)-\psi(0))^{\nu-1}-\frac{1}{\Gamma(\alpha)} \int_{0}^{t} \psi^{\prime}(s)(\psi(t)-\psi(s))^{\alpha-1} \mathfrak{g}(s, \vartheta, \mathfrak{h}(s, \vartheta)) d s\right| \\
& \quad \leq \lambda_{\varphi} \varphi(t, \vartheta) .
\end{aligned}
$$

Hence it follows

$$
\begin{aligned}
|\mathfrak{h}(t, \vartheta)-\mathfrak{y}(t, \vartheta)| & \mid \mathfrak{h}(t, \vartheta)-\frac{\mu(\vartheta)}{\Gamma(v)}(\psi(t)-\psi(0))^{\nu-1} \\
& \quad-\frac{1}{\Gamma(\alpha)} \int_{0}^{t} \psi^{\prime}(s)(\psi(t)-\psi(s))^{\alpha-1} \mathfrak{g}(s, \vartheta, \mathfrak{y}(s, \vartheta)) d s \mid \\
\leq & \mid \mathfrak{h}(t, \vartheta)-\frac{\mu(\vartheta)}{\Gamma(\nu)}(\psi(t)-\psi(0))^{\nu-1} \\
& \quad-\frac{1}{\Gamma(\alpha)} \int_{0}^{t} \psi^{\prime}(s)(\psi(t)-\psi(s))^{\alpha-1} \mathfrak{g}(s, \vartheta, \mathfrak{h}(s, \vartheta)) d s \mid \\
& +\frac{1}{\Gamma(\alpha)} \int_{0}^{t} \psi^{\prime}(s)(\psi(t)-\psi(s))^{\alpha-1}|\mathfrak{g}(s, \vartheta, \mathfrak{h}(s, \vartheta))-\mathfrak{g}(s, \vartheta, \mathfrak{y}(s, \vartheta))| d s \\
\leq & \lambda_{\varphi} \varphi(t, \vartheta)+\frac{\ell(t, \vartheta)}{\Gamma(\alpha)} \int_{0}^{t} \psi^{\prime}(s)(\psi(t)-\psi(s))^{\alpha-1}|\mathfrak{h}(s, \vartheta)-\mathfrak{y}(s, \vartheta)| d s \\
\leq & \lambda_{\varphi} \varphi(t)+\frac{\ell(t, \vartheta)}{\Gamma(\alpha)} \int_{0}^{t} \psi^{\prime}(s)(\psi(t)-\psi(s))^{\alpha-1} \lambda_{\varphi} \varphi(s, \vartheta) d s \\
:= & C_{\mathfrak{g}, \varphi} \varphi(t, \vartheta) .
\end{aligned}
$$

Thus, Eq. (1) is generalized HUR stable.

\section{Illustrative examples}

Here in this part let $\vartheta=(-\infty, 0)$ be equipped with the usual $\sigma$-algebra consisting of Lebesgue measurable subsets of $(-\infty, 0)$. To demonstrate the established theoretical results, $\psi$-HFD is utilized to provide some examples.

Example 4.1 The RFDEs with generalized Riemann-Liouville fractional derivative as particular case of Eq. (1) when $\psi t=t$ is given by

$$
\left\{\begin{array}{l}
\mathscr{D}^{\alpha, \beta ; t} \mathfrak{h}(t, \vartheta)=\mathfrak{g}(t, \vartheta, \mathfrak{h}(t, \vartheta)), \quad t \in[0,1] \\
\left.\ell^{1-v ; t} \mathfrak{h}(t, \vartheta)\right|_{t=0}=\vartheta
\end{array}\right.
$$

Denote $\alpha=\frac{2}{3}, \beta=\frac{1}{2}$ and choose $v=\frac{5}{6}$. Set $\mathfrak{g}(t, \vartheta, \mathfrak{h}(t, \vartheta))=\frac{\vartheta^{2} e^{-t-10}}{1+\vartheta^{2}+|\mathfrak{h}(t, \vartheta)|}$ for $t \in[0,1]$. Moreover, the hypothesis (H1) is satisfied,

$$
|\mathfrak{g}(s, \vartheta, \mathfrak{h}(s, \vartheta))-\mathfrak{g}(s, \vartheta, \mathfrak{y}(s, \vartheta))| \leq \frac{1}{e^{10}}|\mathfrak{h}-\mathfrak{y}|
$$

Finally, the hypothesis (H2) is satisfied with $\varphi(t, \vartheta)=\vartheta^{2} t$ and $\lambda_{\varphi}=\frac{1}{\Gamma(\alpha+2)}$. Thus Eq. (12) is generalized HUR stable. 
Example 4.2 Consider RFDEs with the Hilfer-Hadamard fractional derivative by taking the value $\psi(t)=\ln t$ given by

$$
\left\{\begin{array}{l}
D^{\alpha, \beta ; \ln t} \mathfrak{h}(t, \vartheta)=\frac{1}{9 e^{t}}(\sin |\vartheta| \mathfrak{h}(t, \vartheta)+1), \quad t \in J:=[1, e] \\
\left.\ell^{1-v ; \ln t} \mathfrak{h}(t, \vartheta)\right|_{t=1}=0
\end{array}\right.
$$

Denote $\alpha=\frac{2}{3}, \beta=\frac{1}{2}$ and $\gamma=\frac{5}{6}$. Thus,

$$
|\mathfrak{g}(t, \vartheta, \mathfrak{h}(t, \vartheta))-\mathfrak{g}(t, \vartheta, \mathfrak{y}(t, \vartheta))| \leq \frac{1}{9}|\mathfrak{h}(t, \vartheta)-\mathfrak{y}(t, \vartheta)|
$$

Here $\ell(t, \vartheta)=\frac{1}{9}$. Next, set $\varphi(t, \vartheta)=e^{t+\vartheta}$. Thus we have

$$
\mathfrak{I}^{\alpha ; \ln t} \varphi(t, \vartheta) \leq \frac{1}{\Gamma\left(\frac{5}{3}\right)} \varphi(t, \vartheta)
$$

Thus all the hypotheses are satisfied. Thus Eq. (13) is generalized HUR stable.

Example 4.3 Consider RFDEs involving the Hilfer-Katugampola fractional derivative by taking the value $\psi(t)=t^{\rho}$ given by

$$
\left\{\begin{array}{l}
D^{\alpha, \beta ; t^{\rho}} \mathfrak{h}(t, \vartheta)=\frac{e^{t}}{1+e^{-t}}\left(\frac{\mathfrak{h}(t, \vartheta)}{1+\mathfrak{h}(t, \vartheta)}\right), \quad t \in J:=[0,1] \\
\left.\ell^{1-v ; t^{\rho}} \mathfrak{h}(t, \vartheta)\right|_{t=0}=0
\end{array}\right.
$$

Denote $\alpha=\frac{1}{2}, \beta=\frac{2}{3}$ and $\gamma=\frac{5}{6}$. Thus,

$$
|\mathfrak{g}(t, \vartheta, \mathfrak{h}(t, \vartheta))-\mathfrak{g}(t, \vartheta, \mathfrak{y}(t, \vartheta))| \leq \frac{1}{10}|\mathfrak{h}(t, \vartheta)-\mathfrak{y}(t, \vartheta)|
$$

Finally, set $\varphi(t, \vartheta)=\vartheta^{3} t^{\rho / 2}$. Thus we have

$$
\mathfrak{I}^{\alpha: t^{\rho}} \varphi(t, \vartheta) \leq \frac{1}{\Gamma\left(\frac{3}{2}\right)} \varphi(t, \vartheta)
$$

Thus Eq. (14) is generalized HUR stable.

\section{Conclusion}

In this article, a class of RFDEs has been investigated. By the use of classical fixed point theory of Schauder, we have formed some adequate results as regards the existence of at least one solution to the proposed problem corresponding to HFD. Further an attempt has been made to establish enough conditions for stability analysis. The concerned stability results are devoted to Ulam type which includes UH and HUR stability. The whole analysis has been demonstrated by a suitable example. The respective results are new and interesting to the best of our knowledge regarding stability. As a conclusion the HFD and RFDEs can be used a powerful tools for studying the dynamical behavior of many real world problems. 


\section{Acknowledgements}

We are thankful to the reviewers for their useful corrections and suggestions, which improved the quality of this paper.

\section{Funding}

This research work has been supported financially by the Department of Mathematics, Cankaya University, Ankara, Turkey and Institute of Space Sciences, Bucharest, Romania.

\section{Competing interests}

There is no competing interest corresponding to this paper.

\section{Authors' contributions}

The main idea of this paper was proposed by the first and second authors, while the second and last authors reviewed and modified the paper. Furthermore, all authors read and approved the final manuscript.

\section{Author details}

'Department of Mathematics, Sri Ramakrishna Mission Vidyalaya College of Arts and Science, Coimbatore, India. 2Department of Mathematics, University of Malakand, Chakdara, Pakistan. ${ }^{3}$ Department of Mathematics, Cankaya University, Ankara, Turkey.

\section{Publisher's Note}

Springer Nature remains neutral with regard to jurisdictional claims in published maps and institutional affiliations.

\section{Received: 21 March 2018 Accepted: 15 June 2018 Published online: 26 June 2018}

\section{References}

1. Abbas, S., Benchohra, M., Lazreg, J.-E., N'Guerekata, G.M.: Hilfer and Hadamard functional random fractional differential inclusions. CUBO 19, 17-38 (2017)

2. Abbas, S., Benchohra, M., Sivasundaram, S.: Dynamics and Ulam stability for Hilfer type fractional differential equations. Nonlinear Stud. 4, 627-637 (2016)

3. Ahmad, B., Nieto, J.J.: Riemann-Liouville fractional differential equations with fractional boundary conditions. Fixed Point Theory 13, 329-336 (2013)

4. Almeida, R.: A Caputo fractional derivative of a function with respect to another function. Commun. Nonlinear Sci. Numer. Simul. 44, 460-481 (2017)

5. Furati, K.M., Kassim, M.D., Tatar, N.E.: Existence and uniqueness for a problem involving Hilfer fractional derivative. Comput. Math. Appl. 64, 1616-1626 (2012)

6. Hilfer, R: Application of Fractional Calculus in Physics. World Scientific, Singapore (1999)

7. Ibrahim, R.W.: Generalized Ulam-Hyers stability for fractional differential equations. Int. J. Math. 23, 1-9 (2012)

8. Kamocki, R., Obcznnski, C.: On fractional Cauchy-type problems containing Hilfer derivative. Electron. J. Qual. Theory Differ. Equ. 50, 1 (2016)

9. Kilbas, A.A., Srivastava, H.M., Trujillo, J.J.: Theory and Applications of Fractional Differential Equations. Mathematics Studies, vol. 204. Elsevier, Amsterdam (2006)

10. Kumama, P., Ali, A., Shah, K., Khan, R.A.: Existence results and Hyers-Ulam stability to a class of nonlinear arbitrary order differential equations. J. Nonlinear Sci. Appl. 10, 2986-2997 (2017)

11. Lupulescu, V., Ntouyas, S.K.: Random fractional differential equations. Int. Electron. J. Pure Appl. Math. 4(2), 119-136 (2012)

12. Muniyappan, P., Rajan, S.: Hyers-Ulam-Rassias stability of fractional differential equation. Int. J. Pure Appl. Math. 102, 631-642 (2015)

13. Podlubny, l.: Fractional Differential Equations. Mathematics in Science and Engineering, vol. 198. Academic Press, San Diego (1999)

14. Smart, D.R.: Fixed Point Theorems. Cambridge University Press, Cambridge (1980)

15. Soong, T.T: Random Differential Equations in Science and Engineering. Academic Press, New York (1973)

16. Vanterler da C. Sousa, J., Capelas de Oliveira, E.: On the $\psi$-Hilfer fractional derivative. Commun. Nonlinear Sci. Numer. Simul. 60, 72-91 (2018)

17. Vanterler da C. Sousa, J., Capelas de Oliveira, E.: Ulam-Hyers stability of a nonlinear fractional Volterra integro-differential equation. Appl. Math. Lett. 81, 50-56 (2018).

18. Vanterler da C. Sousa, J., Capelas de Oliveira, E.: On a new operator in fractional calculus and applications. arXiv: 1710.03712

19. Vanterler da C. Sousa, J., Capelas de Oliveira, E.: On the Ulam-Hyers-Rassias stability for nonlinear fractional differential equations using the $\psi$-Hilfer operator. arXiv:1711.07339

20. Vanterler da C. Sousa, J., Capelas de Oliveira, E., Magna, L.A.: Fractional calculus and the ESR test. AIMS Math. 2(4), 692-705 (2017)

21. Vanterler da C. Sousa, J., dos Santos, M.N.N., Magna, L.A., Capelas de Oliveira, E.: Validation of a fractional model for erythrocyte sedimentation rate. arXiv:1802.04340

22. Vivek, D., Kanagarajan, K., Sivasundaram, S.: Dynamics and stability of pantograph equations via Hilfer fractional derivative. Nonlinear Stud. 23, 685-698 (2016)

23. Vu, H.: Random fractional functional differential equations. Int. J. Nonlinear Anal. Appl. 7(2), 253-267 (2016)

24. Wang, J., LV, L., Zhou, Y.: Ulam stability and data dependence for fractional differential equations with Caputo derivative. Electron. J. Qual. Theory Differ. Equ. 63, 1 (2011)

25. Wang, J., Shah, K., Ali, A.: Existence and Hyers-Ulam stability of fractional nonlinear impulsive switched coupled evolution equations. Math. Methods Appl. Sci. 41 2392-2402 (2018) 